DOI 10.14526/02_2018_329

УДК 796.012.22

\title{
ОЦЕНКА ФУНКЦИИ РАВНОВЕСИЯ КАК ИНДИКАТОРА СОСТОЯНИЯ ЗДОРОВЬЯ СТУДЕНТОВ ТЕХНИЧЕСКИХ ВУЗОВ
}

\author{
Булавкина Т.А. ${ }^{1}$, Дубогрызова И.А. ${ }^{1}$, Неклюдова Г.А. ${ }^{2}$ \\ ${ }^{1}$ ФББОУ ВО «Брянский государственный инженерно-технологический университет» \\ Россия, г. Брянск, brfro@yandex.ru \\ - кандидат технических наук \\ ${ }^{2}$ ФГБОУ ВО «Брянский государственный технический университет», \\ Россия, г. Брянск
}

\begin{abstract}
Аннотация. В статье рассматривается возможность экспресс-оценки функиионального состояния студентов на основании оценки статодинамической устойчивости с помощью стабилометрической платформы. Сопоставляются результаты оценки биологического возраста по методике Войтенко и оценки функции равновесия. Материаль. Применение компьютерных стабилографических систем на современном этапе развития технических устройств позволяет эффективно диагностировать состояние здоровья студента. Этот метод относительно прост в использовании, занимает около 4-5 минут для обследования одного человека. Методы: анализ и обобщение научно-методической литературы, педагогический эксперимент, стабилометрия, статистическая проба, динамическая проба, статистическая обработка данных. Результаты. Методика Войтенко использовалась для оценки состояния здоровья в рамках обучения студентов методам самодиагностики, самооценки и самоконтроля своих физиологических возможностей, что является важнейшим принципом выстраивания образовательного пространства кафедры физического воспитания с иелью ориентации студентов на здоровый образ жизни. Определение студентами своего биологического возраста может способствовать их переориентаџии на здоровый образ жизни, являющийся также залогом более успешного обучения профессиональным навыкам. Заключение. Применение стабилографического метода для оценки состояния здоровья по методике Войтенко статистически не подтверждается ( $p \geq 0,05)$, но наблюдается тенденция связи стабилографических показателей и краткосрочного прогноза успешности и результативности физкультурноспортивной деятельности в координационно сложных действиях (спортивные игры). Предполагается, что проведение дальнейших исследований позволит выявить достоверные связи при использовании стабилографического метода в качестве метода для определения состояния здоровья студентов.
\end{abstract}

Ключевье слова: стабилография, состояние функиии равновесия, биологический возраст, состояние здоровья, студенты технических вузов.

Для цитирования: Булавкина Т.А., Дубогрызова И.А., Неклюдова Г.А. Оценка функции равновесия как индикатора состояния здоровья студентов технических вузов. Педагогикопсихологические и медико-биологические проблемы физической культуры и спорта. 2018;. 13(2):224-231. DOI 10.14526/02_2018_329

\section{THE FUNCTION OF BALANCE ESTIMATION AS THE INDICATOR OF STUDENTS' HEALTH STATE AT TECHNICAL HIGHER EDUCATIONAL ESTBALISHMENTS}

\author{
Bulavkina T. A. ${ }^{1}$, Dubogryzova I. A. ${ }^{1}$, Neklyudova G. A. ${ }^{2}$ \\ ${ }^{1}$ Federal State Budgetary Educational Establishment of Higher Education
}




\author{
"Bryansk State Engineering-Technological University" \\ Russia,Bryansk,brfro@yandex.ru \\ ${ }^{2}$ Federal State Budgetary Educational Establishment of Higher Education \\ "Bryansk State Technical University" \\ Russia, Bryansk
}

Annotation. The article is about the opportunity to fulfill express-estimation of students' functional state on the basis of static-dynamic steadiness estimation with the help of stabilometric platform. The results of biological age estimation according to Voytenko methodology and balance function estimation are compared. Materials. Computer stabilographic systems use at a contemporary state of technical devices development helps to diagnose effectively a student's health state. This method is simple enough and one-person examination takes about 4-5 minutes. Research methods: scientific-methodical literature analysis and summarizing, pedagogical experiment, stabilometry, statistical sample, dynamic sample, statistical data handling. Results. Voytenko methodology was used for health state estimation in terms of teaching students the methods of self-diagnostics, selfevaluation and self-control of own physiological abilities, which is the most important principle of educational space organization of physical upbringing department in order to direct students' attention to a healthy life style. Own biological age determination by students provides their redirection to a healthy life style, which is the base of more successful professional skills teaching. Conclusion. Stabilographic method use in order to estimate health state according to Voytenko methodology is not statistically proved $(p \geq 0,05)$, but there is the tendency of stabilographic indices connection with a short-term success prediction and physical-sports activity effectiveness in coordinating difficult actions (sports games). It is supposed that further research works organization will help to reveal valid connections during stabilographic method use as the method for students' health state revelation.

Keywords: posturography, balance function state, biological age, health state, students of technical higher educational establishments.

For citations: Bulavkina T.A., Dubogryzova I.A., Neklyudova G.A. The function of balance estimation as the indicator of students' health state at technical higher educational estbalishments. The Russian Journal of Physical Education and Sport (Pedagogico-Phycological and MedicoBiological Problems of Physical Culture and Sports).2018; 13(2): 224-231. DOI 10.14526/02_2018_329

\section{АКТУАЛЬНОСТЬ ИССЛЕДОВАНИЯ}

Поддержание относительно высокого индивидуального уровня здоровья и психофизической готовности к будущей профессиональной деятельности является одной из основных задач кафедр физического воспитания в технических вузах. Уже давно известно, что оценка функции равновесия человека является индикатором неблагополучия как вестибулярной системы человека, так и интегрального свойства организма состояния здоровья [2,5]. Утомление, интоксикация, заболевания центральной нервной системы проявляют себя в форме расстройств координации в удержании равновесия [5]. Сохранение равновесия - вертикальной позы - человека требует непрерывных движений тела, являющихся результатом взаимодействия высших отделов центральной нервной системы, вестибулярного и зрительного анализаторов, суставной и мышечной проприорецепции. При занятиях физической культурой и спортом способность к освоению техники движений также зависит от уровня развития координационных способностей, в том числе способности сохранять и удерживать равновесие. Ученые считают, что удержание равновесия - динамический феномен, проявляющийся в непрерывном движении тела, которое является результатом взаимодействия 
вестибулярного

и зрительного

анализаторов,

суставно-мышечной

проприорецепции и высших отделов центральной нервной системы [2].

Стабилометрический контроль состояния студентов, занимающихся физическими упражнениями как на основных занятиях физической культурой, так и в целенаправленных секционных занятиях, позволяет оперативно и своевременно выявить тех из них, которые имеют пониженный уровень адаптационных возможностей организма к физическим факторам, а также лиц с начальными симптомами заболеваний («группы риска»).

Целью исследования стало определение соответствия оценки биологического возраста у студентов по методике Войтенко и оценки уровня показателей стабилографии равновесия.

\section{Задачи исследования:}

1. Определить уровень показателя равновесия у студентов технического вуза.

2. Оценить биологический возраст студентов по методике Войтенко.

3. Выявить наличие взаимосвязи между показателями равновесия и уровнем здоровья студентов, определяемых по фактическому биологическому возрасту.

\section{МАТЕРИАЛЫ И МЕТОДЫ}

В качестве основной экспериментальной методики использовалась стабилометрия - метод количественного, пространственного и временного анализа устойчивости поддержания заданной позы человека [3]. Применение компьютерных стабилографических систем на современном этапе развития технических устройств позволяет эффективно диагностировать состояние здоровья студента. Этот метод относительно прост в использовании, занимает около 4-5 минут для обследования одного человека. Краткосрочность процедуры обследования позволяет в условиях учебных занятий по физической культуре обследовать студентов до начала занятия и по окончании этих занятий (до и после получения физической нагрузки).

Используемая в исследовании стабилометрическая платформа «Стабилан-01» применяет компьютеризованные статические двигательно-когнитивные тесты с биологической обратной связью. При проведении исследований были выбраны две стабилографические методики:

1) оценка функции равновесия статистическая проба. Оценка выполнения теста дается в баллах. Это тест типа «Мишень», основанный на комплексной оценке функции равновесия. При выполнении теста требуются точность и дифференцировка положения тела во времени и пространстве. Задача студента заключалась в прямостоянии на стабилографической платформе с концентрацией взгляда на мишени и удержании светового пятна в центре мишени за счет удержания равновесия. Тесты типа «мишень» позволяют оценивать состояние внимания, согласованность зрительного восприятия и мышечного контроля, общую эффективность выполнения целенаправленного действия при удержании неподвижной вертикальной позы;

2) оценка качества управления балансом (статистическая проба) и оценка координации движения (динамическая проба). В этом тесте задействован целый ряд психомоторных проявлений: способность к быстрой и точной реакции на изменение задания, точность и способность к дифференцированию и воспроизведению пространственных и силовых параметров движения (кинестетические способности, основанные на проприоцептивной чувствительности, которые проявляются при необходимости быстро восстанавливать равновесие после смены позы).

Задача студента заключалась в перемещении цветового пятна в различные 
точки экрана за счет микроколебаний туловища стоя на стабилографической платформе. В процессе выполнения пробы компьютером регистрировался стабилографический сигнал, который выдавался в виде оценки в баллах, отнесенной к 3 зонам состояния студента: плохое, среднее и хорошее состояние.

Параллельно с компьютерной стабилографией применялись стандартные тесты, оценивающие физическую подготовленность студентов: бег 100 м (c), бег 3000 м(c), прыжок в длину с места (см), сгибание рук в висе на перекладине (кол-во раз) - для юношей, а также бег 100 м(c), бег 2000 м(c), прыжок в длину с места (см) и сгибание и разгибание туловища в положении лежа на спине (кол-во раз) - для девущек. Результаты тестов физической подготовленности могли переводиться в бальную оценку по сумме 4 тестов.

Проводились антропометрические измерения - определялся рост студентов (см) и их вес (кг).

\section{Дополнительно}

оценивался

биологический возраст студента по методике Войтенко.

Полученные результаты измерений обрабатывались методами математической статистики.

Всего было обследовано 67 студентов и 18 студенток технических вузов 1-го и 2-го курсов, отнесенных к основной медицинской группе. Исследование проводилось на базе кафедр физического воспитания БГИТУ и БГТУ. Обследование проводилось в течение ноября-декабря 2017 г.

\section{РЕЗУЛЬТАТЫ ИССЛЕДОВАНИЯ И ИХ ОБСУЖДЕНИЕ}

При выполнении теста «Мишень» выявлено, что функция равновесия развита плохо у 47\% студентов (муж) и $34 \%$ девушек. Удовлетворительно функция равновесия оценивается у $42 \%$ студентов и у $53 \%$ студенток.Хорошее состояние равновесия выявлено у 7\% студентов (муж) и у 13\% девушек, отличное состояние функции равновесия у девушек не наблюдается (рисунки 1, 2), в то время как такое состояние отмечено у 4\% студентов-юношей. При этом следует отметить, что все студены по медицинским показателям были отнесены к основной группе. По абсолютным средним показателям теста типа «Мишень» среди студентов (мужчин) и студенток (дев) отсутствуют достоверные отличия на 5\% уровне значимости $(\mathrm{p} \geq 0,05)$. У девушек

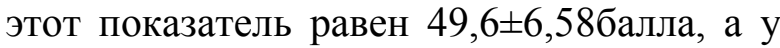
студентов - 40,3 $\pm 2,82$ балла, и эти результаты соответствуют удовлетворительному уровню. На отсутствие половых различий у студентов при выполнении теста «Мишень» указывалось и ранее $[2,3,5]$.

Не выявлено достоверных $(\mathrm{p} \geq 0,05)$ корреляционных связей между результатами теста типа «Мишень», основанного на комплексной оценке функции равновесия, и показателями, входящими в систему оценок биологического возраста по методике Войтенко (задержка дыхания на вдохе (r=$0,22)$, статическая балансировка $(\mathrm{r}=0,09)$, самооценка здоровья $(\mathrm{r}=-0,14)$ и фактический биологический возраст (r=$0,001)$ и весом $(\mathrm{r}=-0,04)$.

В.Е.Косачев [2] в своем исследовании подчеркивал отсутствие статистических связей $\mathrm{c}$ показателями тревожности и личностными свойствами. C помощью факторного анализа автор определил, что стабилографические показатели в его исследовании объединились в два фактора с высоким весом. Причем, кроме стабилографических показателей, в эти факторы не вошли никакие показатели других методик [2].

Методика Войтенко использовалась для оценки состояния здоровья в рамках обучения студентов методам самодиагностики, самооценки и самоконтроля своих физиологических возможностей, что является важнейшим принципом выстраивания образовательного пространства кафедр физического воспитания с целью ориентации студентов на здоровый образ 
жизни. Определение студентами своего биологического возраста может способствовать их переориентации на здоровый образ жизни, являющийся также залогом более успешного обучения профессиональным навыкам.

Сниженные функциональные резервы у молодых людей определяют необходимость применения специальных средств и методов физической культуры на учебных занятиях по физической культуре. Жизнеспособность тела человека определяется не только прожитыми годами, но и степенью износа организма. Пока внутренние органы и системы нормально работают и взаимодействуют друг с другом, поддерживается сбалансированный обмен веществ, происходит обновление старых клеток - организм существует [1]. Календарный (или паспортный) возраст означает, сколько лет прожил человек. Выделяют также понятие биологического возраста. Биологический возраст показывает возраст тела человека (насколько действительно состарилось тело) и обычно не совпадает с календарным возрастом.

Особую практическую значимость данная методика имеет в силу того, что позволяет обратить внимание студентов на самих себя, свое здоровье. Биологический возраст мужчин и женщин рассчитывается по разным формулам [1]:

\section{БВ (биологический возраст)} мужчин:

$$
\begin{aligned}
& 26,985+0,215 \text { АДС - } 0,149 \text { ЗДВ - } \\
& 0,151 \text { СБ }+0,723 \text { СО3 } \\
& \text { БВ } \text { (биологический } \\
& \text { женщин: возраст) } \\
& \text {-1,463 + 0,415 АДП - } 0,140 \text { СБ }+0,248 \\
& \text { МТ + 0,694 СОЗ. } \\
& \text { В формулы расчета входят }
\end{aligned}
$$
следующие показатели:

АДС: (артериальное давление систолическое) в мм.рт.ст.

АДП: (артериальное давление пульсовое). в мм.рт.ст.

3ДВ: (продолжительность задержки дыхания после глубокого вдоха) измеряется трижды с интервалом 5 минут с помощью секундомера. Учитывается наибольшая величина ЗДВ, измеренная в секундах.

СБ: (статическая балансировка, его второе название “японский тест") определяется при стоянии испытуемого на левой ноге, без обуви, глаза закрыты, руки опущены вдоль туловища.

МT: (масса тела в кг).

CO3: (субъективная оценка здоровья) производится с помощью анкеты, включающей 29 вопросов.

Показатели

статической балансировки (“японский тест”) у студентов в среднем имеют очень низкие значения - 30,9 $\pm 9,25$ с, а у студенток $47,4 \pm 11,42$ с. Наблюдается большая величина ошибок среднего арифметического, что обусловлено достаточно малым объемом выборок.

Самооценка здоровья имеет достаточно высокие показатели у студентов (мужчин) 4,2 $\pm 0,45$ балла, а у девушек самооценка гораздо ниже (достоверно $\mathrm{p} \leq 0,05$ ) и составляет $10,6 \pm 1,27$ балла. За время с 2013 года самооценка здоровья у девушек понизилась с $7,3 \pm 0,73[1]$ (достоверно $\mathrm{p} \leq 0,05$ ), что свидетельствует о более плохой самооценке здоровья девушками.

При паспортном возрасте студентов и студенток первого-второго курсов 18-19 лет фактический биологический возраст составил у студенток $27,5 \pm 1,68$ года, а у студентов - 28,1 $\pm 1,86$ года. Такое значительное опережение биологического возраста по отношению к календарному можно интерпретировать как признак снижения уровня здоровья студентов и их функциональных резервов.

$$
\text { У студентов (мужчин) с }
$$
удовлетворительным и плохим уровнем развития функции равновесия выявлены достоверные $(\mathrm{p}<0,05)$ различия по $\mathrm{t}$ критерию Стьюдента только по показателю задержки дыхания на вдохе. 
Все остальные показатели, входящие в формулу определения биологического возраста по методике Войтенко, не имеют достоверных различий $(\mathrm{p} \geq 0,05)$ между студентами, отнесенными к различным категориям по оценке функции равновесия (статическая проба типа «Мишень»).
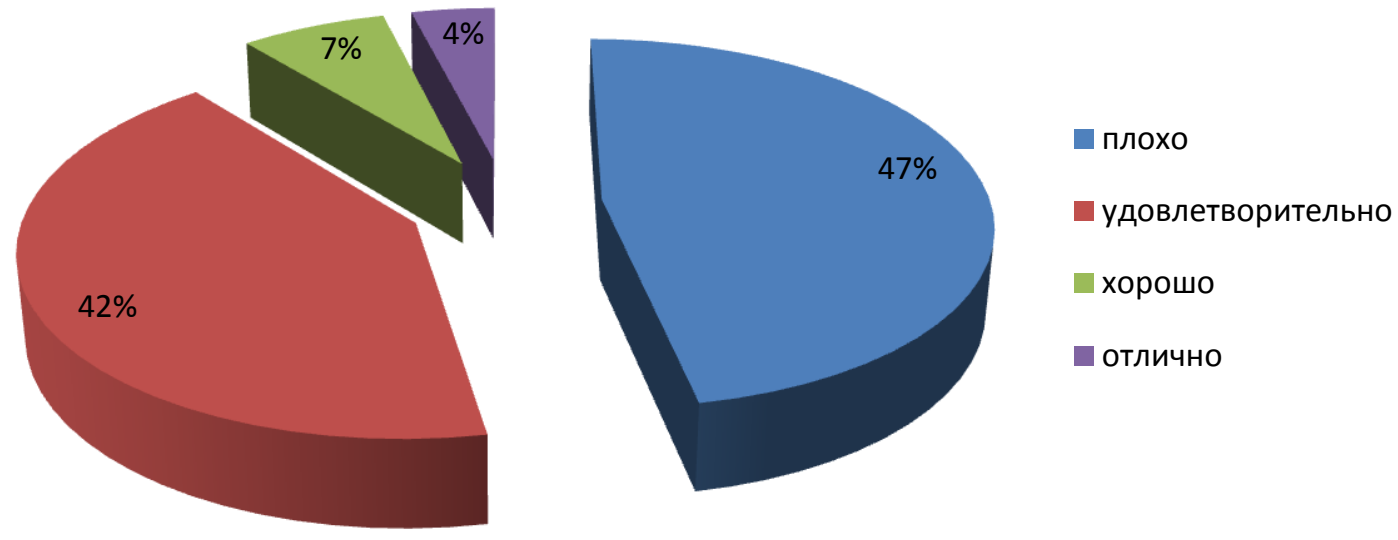

Рисунок 1 - Соотношение уровней развития функции равновесия по стабилографическому показателю теста типа «Мишень» у студентов (мужчин) технических вузов

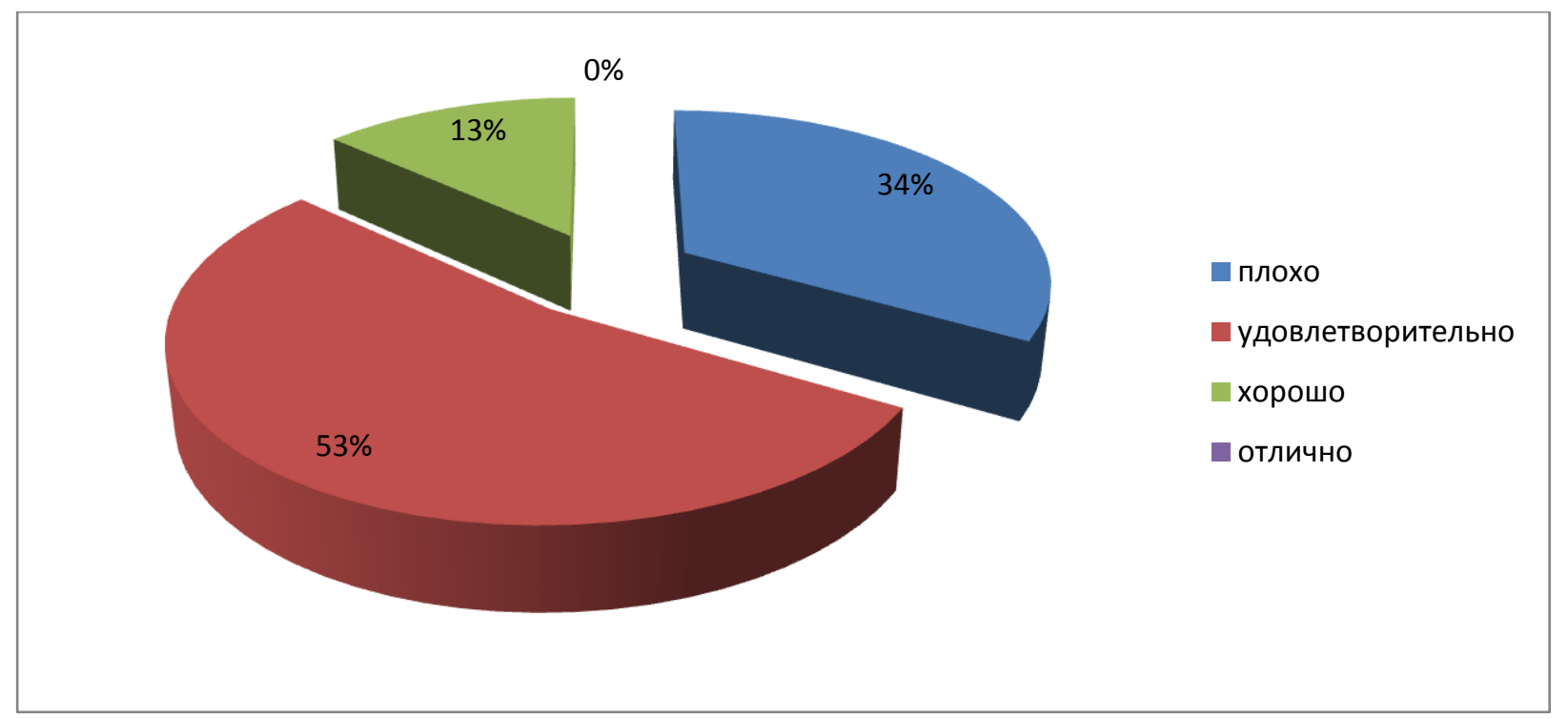

Рисунок 2 - Соотношение уровней развития функции равновесия по стабилографическому показателю теста типа «Мишень» у студенток технических вузов

При сравнении данных студентов и студенток до и после занятий стабилографических обследований физическими упражнениями (1-я и 2 -я 
пробы) отмечается улучшение показателя у девушек в $69 \%$ случаев в различной степени, у мужчин - в 41,7\% случаев. Но проверка этого факта не выявила достоверных различий на $5 \%$ уровне значимости ни у мужчин, ни у девушек.

\section{ЗАКЛЮЧЕНИЕ}

Хороший и отличный уровень развития функции равновесия по стабилографическому показателю теста типа «Мишень» наблюдается только у 13\% девушек и $11 \%$ студентов (мужчин) технических вузов.

Средний биологический возраст студентов составляет $24,4 \pm 0,7$ года при среднем паспортном возрасте $18,3 \pm 0,11$ года. Это свидетельствует о снижении реальных функциональных возможностей организма по сравнению с должными для этого возраста.

Не выявлено достоверных связей между показателями развития функции равновесия на основании применения метода стабилометрии и показателями оценки биологического возраста.

Применение стабилографического метода для оценки состояния здоровья по методике Войтенко статистически не подтверждается $(\mathrm{p} \geq 0,05)$, но наблюдается тенденция связи стабилографических показателей и краткосрочного прогноза успешности и результативности физкультурно-спортивной деятельности в координационно сложных действиях (спортивные игры). Предполагается, что проведение дальнейших исследований позволит выявить достоверные связи при использовании стабилографического метода в качестве метода для определения состояния здоровья студентов.

\section{Список литературы}

1. Булавкина Т.А., Поставнев И.М., Каленикова Н.Г. Биологический возраст студенток как показатель здоровья. Проблемы и перспективы развития физической культуры и спорта в современных условиях: сборник материалов II международной заочной научно-практической конференции. Брянск: Издатель-группа компаний «Десяточка». 2013: 103-106.

2. Косачев В.Е. Стабилография в системе психофизиологического мониторинга. Москва: Известия ТРТУ, Тематический выпуск. 22-24.
3. Кубряк О.В., Гроховский С.С. Практическая стабилометрия. Статические двигательно- когнитивные тесты с биологической обратной связью по опорной реакиии. М.: ООО «ИПЦ «Маска»». 2012: 88.

4. Скворцов Д.В. Стабилометрическое исследование. М.: ООО «ИПЦ „Маска“». 2010: 176.

5. Шестаков М.П., Слива С.С., Войнов И.Д. Компьютерная стабилография в физической культуре и спорте. БИОМЕХАНИКА-2004: тезисы докладов VII Всероссийской конференции по биомеханике: в двух томах. Н.-Новгород. 2004: 188-189.

6. Кузнецова 3.М., Овчинников Ю.Д. Усталость - биомеханическая категория. Педагогико-психологические и медикобиологические проблемы физической культуры и cnopma. 2018; 13(1): 178-183.

7. Kuznetsova Z., Kuznetsov A., Mutaeva I., Khalikov G., Zakharova A., 2015. Athletes training based on a complex assessment of functional state. In Proceedings of the $3^{\text {rd }}$ International Congress on Sport Sciences Research and Technology support. SCITEPRESS. P. 156-160 (Scopus).

8. Kuznetsov A., Mutaeva I., Kuznetsova Z., 2017. Diagnostics of Functional State and Reserve Capacity of young Athletes' Organism. In Proceedings of the $5^{\text {th }}$ International Congress on Sport Sciences Research and Technology support. SCITEPRESS. P. 111-115 (Scopus).

1. Bulavkina T.A., Postavnev I.M., Kalenikova N.G. Biological age of female students as the index of health. Problemy I perspektivy razvitiya fizicheskoj kul'tury I sporta v sovremennyh usloviyah: sbornik materialov II Mezhdunarodnoj zaochnoj nauchno-prakticheskoj konferencii [Problems and prospects of physical culture and sport development in terms of modern conditions: materials digest of the $2^{\text {nd }}$ International extramural scientific-practical conference]. Bryansk: Publisher- groups of companies "Desyatochka". 2013: 103-106.

2. Kosachev V.E. Stabilografiya v sisteme psihofiziologicheskogo monitoringa [Stabilography in the system of psychophysiological monitoring]. Moscow: TRTU News, Thematic issue. 22-24.

3. Kubryak O.V., Grokhovskiy S.S. Prakticheskaya stabilometriya. Staticheskie dvigatel'no-kognitivnye testy s biologicheskoj obratnoj sv'yaz'yu po opornoj reakcii [Practical stabilometry. Static motor-cognitive tests with biological feedback according to support reaction]. Moscow: LLC "Publishing Polygraphic Center "Mask". 2012: 88.

4. Skvortsov D.V. Stabilometricheskoe issledovanie [Stabilometric research]. Moscow: LLC "Publishing Polygraphic Center "Mask"”. 2010: 176.

5. Shestakov M.P., Sliva S.S., Voynov I.D. Computer stabilography in physical culture and sport. BIOMEKHANIKA - 2004: tezisy dokladov VII Vserossijskoj konferencii po biomehanike: $v$ dvuh 
tomah [BIOMECHANICS-2004: theses of reports of the VII All-Russian biomechanics conference: in two volumes]. Nizhni Novgorod. 2004: 188-189.

6. Kuznetsova Z.M., Ovchinnikov Yu.D. Fatigue is a biomechanical category. Pedagogikopsihologicheskie I mediko-biologicheskie problem fizicheskoj kul'tury I sporta = Pedagogicopsychological and medico-biological problems of physical culture and sport. 2018; 13(1): 178-183 [In Russ., In Engl.]

7. Kuznetsova Z., Kuznetsov A., Mutaeva I., Khalikov G., Zakharova A., 2015. Athletes training based on a complex assessment of functional state. In Proceedings of the $3^{\text {rd }}$ International Congress on Sport Sciences Research and Technology support. SCITEPRESS. P. 156-160 (Scopus).

8. Kuznetsov A., Mutaeva I., Kuznetsova Z., 2017. Diagnostics of Functional State and Reserve Capacity of young Athletes' Organism. In Proceedings of the $5^{\text {th }}$ International Congress on Sport Sciences Research and Technology support. SCITEPRESS. P. 111-115 (Scopus).

\title{
Подано: 15.05 .2018
}

Булавкина Татьяна Александровна - дочент, кандидат педагогических наук, доцент кафедры физического воспитания Федеральное государственное бюджетное образовательное учреждение высшего образования «Брянский государственный инженернотехнологический университет», 241037, Россия, г. Брянск, проспект Станке-Димитрова, дом 3, e-mailbrfro@yandex.ru

Дубогрызова Ирина Александровна - доцент, кандидат педагогических наук, доцент кафедры физического воспитания Федеральное государственное бюджетное образовательное учреждение высшего образования «Брянский государственный инженернотехнологический университет», 241037, Россия, г. Брянск, проспект Станке-Димитрова, дом 3

Неклюдова Галина Александровна - дочент, кандидат технических наук, доцент кафедры теоретической механики Федеральное государственное бюджетное образовательное учреждение выстего образования "Брянский государственный технический университет», 241035, Россия, г. Брянск, бульвар 50-летия Октября, дом 7

DOI 10.14526/02_2018_330

УДК 615,82,616.741-009.1

\section{ЭФФЕКТИВНОСТЬ РАННЕЙ ВЕРТИКАЛИЗАЦИИ ПОСЛЕ ОПЕРАТИВНОГО ЛЕЧЕНИЯ ПОЯСНИЧНОГО ОТДЕЛА ПОЗВОНОЧНИКА}

\author{
Вадутов Р.Р. ${ }^{1}$ Быков Е.В. ${ }^{1}$, Коломиец О.И. ${ }^{1}$ \\ ${ }^{1}$ ФГБОУ ВО «Уральский государственный университет физической культурыл», \\ Россия, г. Челябинск, 2677489@таil.ru
}

\begin{abstract}
Аннотация: ускорение восстановительных проиессов является основной задачей реабилитации в постопераџионный период. В стандарт реабилитации после хирургической фиксации ПОП входит ЛФК, массаж и физиотерапия, что в послеоперационньй период восстановления часто вызывает временное увеличение боли и отека. Возникает чувство страха вертикализации и физической активности. Подобные симптомы предложено нивелировать предварительным крио-массажем [19]. Материалы. 30 пациентовспортсменов в нейрохирургическом отделении Свердловского областного клинического госпиталя (16 мужчин и 14 женщин в возрасте 24士4,2 года) после оперативного лечения
\end{abstract}

